\title{
On a more accurate multidimensional Mulholland-type inequality
}

Qiang Chen ${ }^{1}$ and Bicheng Yang ${ }^{2^{*}}$

\section{"Correspondence:}

bcyang@gdei.edu.cn;

bcyang818@163.com

${ }^{2}$ Department of Mathematics,

Guangdong University of

Education, Guangzhou, Guangdong

510303, P.R. China

Full list of author information is

available at the end of the article

\begin{abstract}
In this paper, by using the way of weight coefficients and technique of real analysis, a more accurate multidimensional discrete Mulholland-type inequality with the best possible constant factor is given, which is an extension of the Mulholland inequality. The equivalent form, the operator expression with the norm as well as a few particular cases are also considered.
\end{abstract}

MSC: 26D15; 47A07

Keywords: Mulholland-type inequality; weight coefficient; equivalent form; operator; norm

\section{Introduction}

Suppose that $p>1, \frac{1}{p}+\frac{1}{q}=1, f(x), g(y) \geq 0, f \in L^{p}\left(\mathbf{R}_{+}\right), g \in L^{q}\left(\mathbf{R}_{+}\right),\|f\|_{p}=\left\{\int_{0}^{\infty} f^{p}(x) d x\right\}^{\frac{1}{p}}>$ $0,\|g\|_{q}>0$. We have the following Hardy-Hilbert integral inequality (cf. [1]):

$$
\int_{0}^{\infty} \int_{0}^{\infty} \frac{f(x) g(y)}{x+y} d x d y<\frac{\pi}{\sin (\pi / p)}\|f\|_{p}\|g\|_{q}
$$

where the constant factor $\frac{\pi}{\sin (\pi / p)}$ is the best possible. Assuming that $a_{m}, b_{n} \geq 0, a=$ $\left\{a_{m}\right\}_{m=1}^{\infty} \in l^{p}, b=\left\{b_{n}\right\}_{n=1}^{\infty} \in l^{q},\|a\|_{p}=\left\{\sum_{m=1}^{\infty} a_{m}^{p}\right\}^{\frac{1}{p}}>0,\|b\|_{q}>0$, we have the following Hardy-Hilbert inequality with the same best constant $\frac{\pi}{\sin (\pi / p)}(c f .[1])$ :

$$
\sum_{m=1}^{\infty} \sum_{n=1}^{\infty} \frac{a_{m} b_{n}}{m+n}<\frac{\pi}{\sin (\pi / p)}\|a\|_{p}\|b\|_{q}
$$

Inequalities (1) and (2) are important in analysis and its applications (cf. [1-6]). Also we have the following Mulholland inequality (cf. [1]):

$$
\sum_{m=2}^{\infty} \sum_{n=2}^{\infty} \frac{a_{m} b_{n}}{\ln m n}<\frac{\pi}{\sin (\pi / p)}\left\{\sum_{m=2}^{\infty} \frac{a_{m}^{p}}{m^{1-p}}\right\}^{\frac{1}{p}}\left\{\sum_{n=2}^{\infty} \frac{b_{n}^{q}}{n^{1-q}}\right\}^{\frac{1}{q}} .
$$

In 1998, by introducing an independent parameter $\lambda \in(0,1]$, Yang [7] gave an extension of (1) for $p=q=2$. Yang [5] gave some extensions of (1) and (2) as follows: If $\lambda_{1}, \lambda_{2}, \lambda \in \mathbf{R}$, $\lambda_{1}+\lambda_{2}=\lambda, k_{\lambda}(x, y)$ is a non-negative homogeneous function of degree $-\lambda$, with

$$
k\left(\lambda_{1}\right)=\int_{0}^{\infty} k_{\lambda}(t, 1) t^{\lambda_{1}-1} d t \in \mathbf{R}_{+},
$$

C 2014 Chen and Yang; licensee Springer. This is an Open Access article distributed under the terms of the Creative Commons Attribution License (http://creativecommons.org/licenses/by/2.0), which permits unrestricted use, distribution, and reproduction in any medium, provided the original work is properly cited. 


$$
\begin{aligned}
& \phi(x)=x^{p\left(1-\lambda_{1}\right)-1}, \psi(x)=x^{q\left(1-\lambda_{2}\right)-1}, f(x), g(y) \geq 0, \\
& f \in L_{p, \phi}\left(\mathbf{R}_{+}\right)=\left\{f ;\|f\|_{p, \phi}:=\left\{\int_{0}^{\infty} \phi(x)|f(x)|^{p} d x\right\}^{\frac{1}{p}}<\infty\right\}, \\
& g \in L_{q, \psi}\left(\mathbf{R}_{+}\right),\|f\|_{p, \phi},\|g\|_{q, \psi}>0 \text {, then } \\
& \int_{0}^{\infty} \int_{0}^{\infty} k_{\lambda}(x, y) f(x) g(y) d x d y<k\left(\lambda_{1}\right)\|f\|_{p, \phi}\|g\|_{q, \psi},
\end{aligned}
$$

where the constant factor $k\left(\lambda_{1}\right)$ is the best possible. Moreover, if $k_{\lambda}(x, y)$ is finite and $k_{\lambda}(x, y) x^{\lambda_{1}-1}\left(k_{\lambda}(x, y) y^{\lambda_{2}-1}\right)$ is decreasing with respect to $x>0(y>0)$, then for $a_{m}, b_{n} \geq 0$,

$$
a \in l_{p, \phi}=\left\{a ;\|a\|_{p, \phi}:=\left\{\sum_{n=1}^{\infty} \phi(n)\left|a_{n}\right|^{p}\right\}^{\frac{1}{p}}<\infty\right\},
$$

$b=\left\{b_{n}\right\}_{n=1}^{\infty} \in l_{q, \psi},\|a\|_{p, \phi},\|b\|_{q, \psi}>0$, it follows that

$$
\sum_{m=1}^{\infty} \sum_{n=1}^{\infty} k_{\lambda}(m, n) a_{m} b_{n}<k\left(\lambda_{1}\right)\|a\|_{p, \phi}\|b\|_{q, \psi},
$$

where the constant factor $k\left(\lambda_{1}\right)$ is still the best possible.

Clearly, for $\lambda=1, k_{1}(x, y)=\frac{1}{x+y}, \lambda_{1}=\frac{1}{q}, \lambda_{2}=\frac{1}{p}$, inequality (3) reduces to (1), while (5) reduces to (2). Some other results including the multidimensional Hilbert-type integral inequalities are provided by [8-24].

About half-discrete Hilbert-type inequalities with the non-homogeneous kernels, Hardy et al. provided a few results in Theorem 351 of [1]. But they did not prove that the constant factors are the best possible. However, Yang [25] gave a result with the kernel $\frac{1}{(1+n x)^{\lambda}}$ $(0<\lambda \leq 2)$ by introducing a variable and proved that the constant factor is the best possible. In 2011 Yang [26] gave a half-discrete Hardy-Hilbert inequality with the best possible constant factor. Zhong et al. [27-33] investigated several half-discrete Hilbert-type inequalities with particular kernels. Using the way of weight functions and the techniques of discrete and integral Hilbert-type inequalities with some additional conditions on the kernel, a half-discrete Hilbert-type inequality with a general homogeneous kernel of degree $-\lambda \in \mathbf{R}$ and a best constant factor $k\left(\lambda_{1}\right)$ is obtained as follows:

$$
\int_{0}^{\infty} f(x) \sum_{n=1}^{\infty} k_{\lambda}(x, n) a_{n} d x<k\left(\lambda_{1}\right)\|f\|_{p, \phi}\|a\|_{q, \psi}
$$

(see Yang and Chen [34]). At the same time, a half-discrete Hilbert-type inequality with a general non-homogeneous kernel and the best constant factor is given by Yang [35].

In this paper, by using the way of weight coefficients and technique of real analysis, a more accurate multidimensional discrete Mulholland-type inequality with the best possible constant factor is given, which is an extension of (3). The equivalent form, the operator expression with the norm as well as a few particular cases are also considered. 


\section{Some lemmas}

Lemma 1 If $(-1)^{i} h^{(i)}(t)>0(t>0 ; i=1,2)$, then for $b>0,0<\alpha \leq 1$,

$$
(-1)^{i} \frac{d^{i}}{d x^{i}} h\left(\left(b+\ln ^{\alpha} x\right)^{\frac{1}{\alpha}}\right)>0 \quad(x>1 ; i=1,2) .
$$

Proof We find

$$
\begin{aligned}
\frac{d}{d x} h\left(\left(b+\ln ^{\alpha} x\right)^{\frac{1}{\alpha}}\right)= & \frac{1}{x} h^{\prime}\left(\left(b+\ln ^{\alpha} x\right)^{\frac{1}{\alpha}}\right)\left(b+\ln ^{\alpha} x\right)^{\frac{1}{\alpha}-1} \ln ^{\alpha-1} x<0, \\
\frac{d^{2}}{d x^{2}} h\left(\left(b+\ln ^{\alpha} x\right)^{\frac{1}{\alpha}}\right)= & \frac{d}{d x}\left[\frac{1}{x} h^{\prime}\left(\left(b+\ln ^{\alpha} x\right)^{\frac{1}{\alpha}}\right)\left(b+\ln ^{\alpha} x\right)^{\frac{1}{\alpha}-1} \ln ^{\alpha-1} x\right] \\
= & -\frac{1}{x^{2}} h^{\prime}\left(\left(b+\ln ^{\alpha} x\right)^{\frac{1}{\alpha}}\right)\left(b+\ln ^{\alpha} x\right)^{\frac{1}{\alpha}-1} \ln ^{\alpha-1} x \\
& +\frac{1}{x^{2}} h^{\prime \prime}\left(\left(b+\ln ^{\alpha} x\right)^{\frac{1}{\alpha}}\right)\left(b+\ln ^{\alpha} x\right)^{\frac{2}{\alpha}-2} \ln ^{2 \alpha-2} x \\
& +\alpha\left(\frac{1}{\alpha}-1\right) \frac{1}{x^{2}} h^{\prime}\left(\left(b+\ln ^{\alpha} x\right)^{\frac{1}{\alpha}}\right)\left(b+\ln ^{\alpha} x\right)^{\frac{1}{\alpha}-2} \ln ^{2 \alpha-2} x \\
& +(\alpha-1) \frac{1}{x^{2}} h^{\prime}\left(\left(b+\ln ^{\alpha} x\right)^{\frac{1}{\alpha}}\right)\left(b+\ln ^{\alpha} x\right)^{\frac{1}{\alpha}-1} \ln ^{\alpha-2} x \\
= & {\left[-h^{\prime}\left(\left(b+\ln ^{\alpha} x\right)^{\frac{1}{\alpha}}\right)\left(b+\ln ^{\alpha} x\right) \ln ^{2} x\right.} \\
& +h^{\prime \prime}\left(\left(b+\ln ^{\alpha} x\right)^{\frac{1}{\alpha}}\right)\left(b+\ln ^{\alpha} x\right)^{\frac{1}{\alpha}} \ln ^{\alpha} x \\
& \left.+b(\alpha-1) h^{\prime}\left(\left(b+\ln ^{\alpha} x\right)^{\frac{1}{\alpha}}\right)\right] \frac{1}{x^{2}}\left(b+\ln ^{\alpha} x\right)^{\frac{1}{\alpha}-2} \ln ^{\alpha-2} x>0 .
\end{aligned}
$$

Then we have (7).

If $i_{0}, j_{0} \in \mathbf{N}$ ( $\mathbf{N}$ is the set of positive integers), $\alpha, \beta>0$, we set

$$
\begin{aligned}
& \|x\|_{\alpha}:=\left(\sum_{k=1}^{i_{0}}\left|x_{k}\right|^{\alpha}\right)^{\frac{1}{\alpha}} \quad\left(x=\left(x_{1}, \ldots, x_{i_{0}}\right) \in \mathbf{R}^{i_{0}}\right), \\
& \|y\|_{\beta}:=\left(\sum_{k=1}^{j_{0}}\left|y_{k}\right|^{\beta}\right)^{\frac{1}{\beta}} \quad\left(y=\left(y_{1}, \ldots, y_{j_{0}}\right) \in \mathbf{R}^{j_{0}}\right) .
\end{aligned}
$$

Lemma 2 If $s \in \mathbf{N}, \gamma, M>0, \Psi(u)$ is a non-negative measurable function in $(0,1]$, and

$$
D_{M}:=\left\{x \in \mathbf{R}_{+}^{s} ; \sum_{i=1}^{s} x_{i}^{\gamma} \leq M^{\gamma}\right\}=\left\{x ; \sum_{i=1}^{s}\left(\frac{x_{i}}{M}\right)^{\gamma} \leq 1\right\},
$$

then we have (cf. [36])

$$
\begin{gathered}
\int \cdots \int_{D_{M}} \Psi\left(\sum_{i=1}^{s}\left(\frac{x_{i}}{M}\right)^{\gamma}\right) d x_{1} \cdots d x_{s} \\
=\frac{M^{s} \Gamma^{s}\left(\frac{1}{\gamma}\right)}{\gamma^{s} \Gamma\left(\frac{s}{\gamma}\right)} \int_{0}^{1} \Psi(u) u^{\frac{s}{\gamma}-1} d u .
\end{gathered}
$$


Lemma 3 If $s \in \mathbf{N}, \gamma>0, \varepsilon>0, d=\left(d_{1}, \ldots, d_{s}\right) \in\left[\frac{1}{2}, 1\right]^{s}$, then

$$
\begin{aligned}
A_{s}(\varepsilon) & :=\sum_{m}\|\ln (m+d)\|_{\gamma}^{-s-\varepsilon} \frac{1}{\prod_{i=1}^{s}\left(m_{i}+d_{i}\right)} \\
& =\frac{\Gamma^{s}\left(\frac{1}{\gamma}\right)}{\varepsilon S^{\varepsilon / \gamma} \gamma^{s-1} \Gamma\left(\frac{s}{\gamma}\right)}+O(1) \quad\left(\varepsilon \rightarrow 0^{+}\right) .
\end{aligned}
$$

Proof For $M>s^{1 / \gamma}$, we set

$$
\Psi(u)= \begin{cases}0, & 0<u<\frac{s}{M^{\gamma}}, \\ \left(M u^{1 / \gamma}\right)^{-s-\varepsilon}, & \frac{s}{M^{\gamma}} \leq u \leq 1 .\end{cases}
$$

Then by the decreasing property and (10), it follows that

$$
\begin{aligned}
& A_{s}(\varepsilon) \geq \int_{\left\{x \in \mathbf{R}_{+}^{s} ; x_{i} \geq e-d_{i}\right\}}\|\ln (x+d)\|_{\gamma}^{-s-\varepsilon} \frac{d x}{\prod_{i=1}^{s}\left(x_{i}+d_{i}\right)} \\
& \stackrel{u_{i}=\ln \left(x_{i}+d_{i}\right)}{=} \int_{\left\{u \in \mathbf{R}_{+}^{s} ; u_{i} \geq 1\right\}}\|u\|_{\gamma}^{-s-\varepsilon} d u \\
& \quad=\lim _{M \rightarrow \infty} \int \cdots \int_{D_{M}} \Psi\left(\sum_{i=1}^{s}\left(\frac{x_{i}}{M}\right)^{\gamma}\right) d x_{1} \cdots d x_{s} \\
&=\lim _{M \rightarrow \infty} \frac{M^{s} \Gamma^{s}\left(\frac{1}{\gamma}\right)}{\gamma^{s} \Gamma\left(\frac{s}{\gamma}\right)} \int_{s / M \gamma}^{1}\left(M u^{1 / \gamma}\right)^{-s-\varepsilon} u^{\frac{s}{\gamma}-1} d u=\frac{\Gamma^{s}\left(\frac{1}{\gamma}\right)}{\varepsilon s^{\varepsilon / \gamma} \gamma^{s-1} \Gamma\left(\frac{s}{\gamma}\right)} .
\end{aligned}
$$

In the following, by mathematical induction we prove that, for any $s \in \mathbf{N}$,

$$
A_{s}(\varepsilon) \leq O_{s}(1)+\frac{\Gamma^{s}\left(\frac{1}{\gamma}\right)}{\varepsilon s^{\varepsilon / \gamma} \gamma^{s-1} \Gamma\left(\frac{s}{\gamma}\right)} \quad\left(\varepsilon \rightarrow 0^{+}\right)
$$

For $s=1$, by the Hermite-Hadamard inequality ( $c f$. [37]), it follows that

$$
\begin{aligned}
A_{1}(\varepsilon) & =\sum_{m_{1}=1}^{2} \frac{\ln ^{-1-\varepsilon}\left(m_{1}+d_{1}\right)}{m_{1}+d_{1}}+\sum_{m_{1}=3}^{\infty} \frac{\ln ^{-1-\varepsilon}\left(m_{1}+d_{1}\right)}{m_{1}+d_{1}} \\
& \leq O_{1}(1)+\int_{\frac{5}{2}}^{\infty} \frac{\ln ^{-1-\varepsilon}\left(x+d_{1}\right) d x}{x+d_{1}} \leq O_{1}(1)+\int_{e-d_{1}}^{\infty} \frac{\ln ^{-1-\varepsilon}\left(x+d_{1}\right) d x}{x+d_{1}} \\
& \stackrel{u=\ln \left(x+d_{1}\right)}{=} O_{1}(1)+\int_{1}^{\infty} u^{-1-\varepsilon} d u=O_{1}(1)+\frac{1}{\varepsilon},
\end{aligned}
$$

and then (12) is valid. Assuming that (12) is valid for $s-1 \in \mathbf{N}$, then for $s$, we set

$$
\begin{aligned}
A_{s}(\varepsilon)= & \sum_{\left\{m \in \mathbf{N}^{s} ; \exists i_{0}, m_{i_{0}}=1,2\right\}}\|\ln (m+d)\|_{\gamma}^{-s-\varepsilon} \frac{1}{\prod_{i=1}^{s}\left(m_{i}+d_{i}\right)} \\
& +\sum_{\left\{m \in \mathbf{N}^{s} ; m_{i} \geq 3\right\}}\|\ln (m+d)\|_{\gamma}^{-s-\varepsilon} \frac{1}{\prod_{i=1}^{s}\left(m_{i}+d_{i}\right)} .
\end{aligned}
$$


There exist constants $a, b \in \mathbf{R}_{+}$, such that

$$
\begin{aligned}
& \sum_{\left\{m \in \mathbf{N}^{\left.s ; \exists i_{0}, m_{i_{0}}=1,2\right\}}\right.}\|\ln (m+d)\|_{\gamma}^{-s-\varepsilon} \frac{1}{\prod_{i=1}^{s}\left(m_{i}+d_{i}\right)} \\
& \leq a+b \sum_{\left\{m \in \mathbf{N}^{s-1} ; m_{i} \geq 1\right\}}\|\ln (m+d)\|_{\gamma}^{-(s-1)-(1+\varepsilon)} \frac{1}{\prod_{i=1}^{s-1}\left(m_{i}+d_{i}\right)} .
\end{aligned}
$$

By the assumption of mathematical induction for $s-1$, we find

$$
\begin{aligned}
& \sum_{\left\{m \in \mathbf{N}^{s-1} ; m_{i} \geq 1\right\}}\|\ln (m+d)\|_{\gamma}^{-(s-1)-(1+\varepsilon)} \frac{1}{\prod_{i=1}^{s-1}\left(m_{i}+d_{i}\right)} \\
& \leq O_{s-1}(1)+\frac{\Gamma^{s-1}\left(\frac{1}{\gamma}\right)}{(1+\varepsilon)(s-1)^{(1+\varepsilon) / \gamma} \gamma^{s-2} \Gamma\left(\frac{s-1}{\gamma}\right)},
\end{aligned}
$$

and then

$$
\sum_{\left\{m \in \mathbf{N}^{s} ; \exists i_{0}, m_{i_{0}}=1,2\right\}}\|\ln (m+d)\|_{\gamma}^{-s-\varepsilon} \frac{1}{\prod_{i=1}^{s}\left(m_{i}+d_{i}\right)} \leq O_{s}(1) .
$$

By Lemma 1 and the Hermite-Hadamard inequality (cf. [37]), we obtain

$$
\begin{aligned}
& \sum_{\left\{m \in \mathbf{N}^{s} ; m_{i} \geq 3\right\}}\|\ln (m+d)\|_{\gamma}^{-s-\varepsilon} \frac{1}{\prod_{i=1}^{s}\left(m_{i}+d_{i}\right)} \\
& \leq \int_{\left\{x \in \mathbf{R}_{+}^{s} ; x_{i} \geq \frac{5}{2}\right\}}\|\ln (x+d)\|_{\gamma}^{-s-\varepsilon} \frac{1}{\prod_{i=1}^{s}\left(x_{i}+d_{i}\right)} d x \\
& \leq \int_{\left\{x \in \mathbf{R}_{+}^{s} ; x_{i} \geq e-d_{i}\right\}}\|\ln (x+d)\|_{\gamma}^{-s-\varepsilon} \frac{1}{\prod_{i=1}^{s}\left(x_{i}+d_{i}\right)} d x \\
& u_{i=\ln \left(x_{i}+d_{i}\right)} \int_{\left\{u \in \mathbf{R}_{+}^{s} ; u_{i} \geq 1\right\}}\|u\|_{\gamma}^{-s-\varepsilon} d u=\frac{\Gamma^{s}\left(\frac{1}{\gamma}\right)}{\varepsilon s^{\varepsilon / \gamma} \gamma^{s-1} \Gamma\left(\frac{s}{\gamma}\right)} .
\end{aligned}
$$

Hence we prove that (12) is valid for $s \in \mathbf{N}$. Therefore, we have (11).

Lemma 4 If $\mathbf{C}$ is the set of complex numbers and $\mathbf{C}_{\infty}=\mathbf{C} \cup\{\infty\}, z_{k} \in \mathbf{C} \backslash\{z \mid \operatorname{Re} z \geq 0, \operatorname{Im} z=$ $0\}(k=1,2, \ldots, n)$ are different points, the function $f(z)$ is analytic in $\mathbf{C}_{\infty}$ except for $z_{i}(i=$ $1,2, \ldots, n)$, and $z=\infty$ is a zero point of $f(z)$ whose order is not less than 1 , then for $\alpha \in \mathbf{R}$, we have

$$
\int_{0}^{\infty} f(x) x^{\alpha-1} d x=\frac{2 \pi i}{1-e^{2 \pi \alpha i}} \sum_{k=1}^{n} \operatorname{Re} s\left[f(z) z^{\alpha-1}, z_{k}\right]
$$

where $0<\operatorname{Im} \ln z=\arg z<2 \pi$. In particular, if $z_{k}(k=1, \ldots, n)$ are all poles of order 1 , setting $\varphi_{k}(z)=\left(z-z_{k}\right) f(z)\left(\varphi_{k}\left(z_{k}\right) \neq 0\right)$, then

$$
\int_{0}^{\infty} f(x) x^{\alpha-1} d x=\frac{\pi}{\sin \pi \alpha} \sum_{k=1}^{n}\left(-z_{k}\right)^{\alpha-1} \varphi_{k}\left(z_{k}\right) .
$$


Proof By [38, p.118], we have (13). We find

$$
\begin{aligned}
1-e^{2 \pi \alpha i} & =1-\cos 2 \pi \alpha-i \sin 2 \pi \alpha \\
& =-2 i \sin \pi \alpha(\cos \pi \alpha+i \sin \pi \alpha)=-2 i e^{i \pi \alpha} \sin \pi \alpha .
\end{aligned}
$$

In particular, since $f(z) z^{\alpha-1}=\frac{1}{z-z_{k}}\left(\varphi_{k}(z) z^{\alpha-1}\right)$, it is obvious that

$$
\operatorname{Re} s\left[f(z) z^{\alpha-1},-a_{k}\right]=z_{k}^{\alpha-1} \varphi_{k}\left(z_{k}\right)=-e^{i \pi \alpha}\left(-z_{k}\right)^{\alpha-1} \varphi_{k}\left(z_{k}\right) .
$$

Then by (13), we obtain (14).

Example 1 For $s \in \mathbf{N}$, we set

$$
k_{\lambda}(x, y)=\prod_{k=1}^{s} \frac{1}{\left(x^{\lambda / s}+c_{k} y^{\lambda / s}\right)} \quad\left(0<c_{1}<\cdots<c_{s}, 0<\lambda \leq s\right) .
$$

For $0<\lambda_{1} \leq i_{0}, 0<\lambda_{2} \leq j_{0}, \lambda_{1}+\lambda_{2}=\lambda$, by (14), we find

$$
\begin{aligned}
& k_{s}\left(\lambda_{1}\right):=\int_{0}^{\infty} \prod_{k=1}^{s} \frac{1}{t^{\lambda / s}+c_{k}} t^{\lambda_{1}-1} d t \\
& \stackrel{u=t^{\lambda / s}}{=} \frac{s}{\lambda} \int_{0}^{\infty} \prod_{k=1}^{s} \frac{1}{u+c_{k}} u^{\frac{s \lambda_{1}}{\lambda}-1} d u \\
& =\frac{\pi s}{\lambda \sin \left(\frac{\pi s \lambda_{1}}{\lambda}\right)} \sum_{k=1}^{s} c_{k}^{\frac{s \lambda_{1}}{\lambda}-1} \prod_{j=1(j \neq k)}^{s} \frac{1}{c_{j}-c_{k}} \in \mathbf{R}_{+} .
\end{aligned}
$$

In particular, for $s=1$, we obtain

$$
k_{1}\left(\lambda_{1}\right)=\frac{1}{\lambda} \int_{0}^{\infty} \frac{u^{\left(\lambda_{1} / \lambda\right)-1}}{u+c_{1}} d u=\frac{\pi}{\lambda \sin \left(\frac{\pi \lambda_{1}}{\lambda}\right)} c_{1}^{\frac{\lambda_{1}}{\lambda}-1}
$$

Definition 1 For $s \in \mathbf{N}, 0<\alpha, \beta \leq 1,0<c_{1}<\cdots<c_{s}, 0<\lambda \leq s, 0<\lambda_{1} \leq i_{0}, 0<\lambda_{2} \leq$ $j_{0}, \lambda_{1}+\lambda_{2}=\lambda, \tau=\left(\tau_{1}, \ldots, \tau_{i_{0}}\right) \in\left[\frac{1}{2}, 1\right]^{i_{0}}, \sigma=\left(\sigma_{1}, \ldots, \sigma_{j_{0}}\right) \in\left[\frac{1}{2}, 1\right]^{j_{0}}, \ln (m+\tau)=\left(\ln \left(m_{1}+\right.\right.$ $\left.\left.\tau_{1}\right), \ldots, \ln \left(m_{i_{0}}+\tau_{i_{0}}\right)\right) \in \mathbf{R}_{+}^{i_{0}}, \ln (n+\sigma)=\left(\ln \left(n_{1}+\sigma_{1}\right), \ldots, \ln \left(n_{j_{0}}+\sigma_{j_{0}}\right)\right) \in \mathbf{R}_{+}^{j_{0}}$, we define two weight coefficients $w_{\lambda}\left(\lambda_{2}, n\right)$ and $W_{\lambda}\left(\lambda_{1}, m\right)$ as follows:

$$
\begin{aligned}
& w_{\lambda}\left(\lambda_{2}, n\right):=\sum_{m} \frac{\|\ln (n+\sigma)\|_{\beta}^{\lambda_{2}}\|\ln (m+\tau)\|_{\alpha}^{\lambda_{1}-i_{0}}}{\prod_{k=1}^{s}\left[\|\ln (m+\tau)\|_{\alpha}^{\lambda / s}+c_{k}\|\ln (n+\sigma)\|_{\beta}^{\lambda / s}\right] \prod_{i=1}^{i_{0}}\left(m_{i}+\tau_{i}\right)}, \\
& W_{\lambda}\left(\lambda_{1}, m\right):=\sum_{n} \frac{\|\ln (m+\tau)\|_{\alpha}^{\lambda_{1}}\|\ln (n+\sigma)\|_{\beta}^{\lambda_{2}-j_{0}}}{\prod_{k=1}^{s}\left[\|\ln (m+\tau)\|_{\alpha}^{\lambda / s}+c_{k}\|\ln (n+\sigma)\|_{\beta}^{\lambda / s}\right] \prod_{j=1}^{j_{0}}\left(n_{j}+\sigma_{j}\right)},
\end{aligned}
$$

where $\sum_{m}=\sum_{m_{i_{0}}=1}^{\infty} \cdots \sum_{m_{1}=1}^{\infty}$ and $\sum_{n}=\sum_{n_{j_{0}}=1}^{\infty} \cdots \sum_{n_{1}=1}^{\infty}$.

Lemma 5 Let the assumptions as in Definition 1 be fulfilled. Then: 
(i) we have

$$
\begin{aligned}
& w_{\lambda}\left(\lambda_{2}, n\right)<K_{2} \quad\left(n \in \mathbf{N}^{j_{0}}\right), \\
& W_{\lambda}\left(\lambda_{1}, m\right)<K_{1} \quad\left(m \in \mathbf{N}^{i_{0}}\right),
\end{aligned}
$$

where

$$
K_{1}:=\frac{\Gamma^{j_{0}}\left(\frac{1}{\beta}\right)}{\beta^{j_{0}-1} \Gamma\left(\frac{j_{0}}{\beta}\right)} k_{s}\left(\lambda_{1}\right), \quad K_{2}:=\frac{\Gamma^{i_{0}}\left(\frac{1}{\alpha}\right)}{\alpha^{i_{0}-1} \Gamma\left(\frac{i_{0}}{\alpha}\right)} k_{s}\left(\lambda_{1}\right)
$$

and $k_{s}\left(\lambda_{1}\right)$ is indicated by (15);

(ii) for $p>1,0<\varepsilon<p \min \left\{\lambda_{1}, 1-\lambda_{2}\right\}$, setting $\tilde{\lambda}_{1}=\lambda_{1}-\frac{\varepsilon}{p}, \tilde{\lambda}_{2}=\lambda_{2}+\frac{\varepsilon}{p}$, we have

$$
0<\widetilde{K}_{2}\left(1-\widetilde{\theta}_{\lambda}(n)\right)<w_{\lambda}\left(\widetilde{\lambda}_{2}, n\right)
$$

where

$$
\begin{aligned}
\widetilde{\theta}_{\lambda}(n) & :=\frac{1}{k_{s}\left(\widetilde{\lambda}_{1}\right)} \int_{0}^{i_{0}^{\lambda /(\alpha s)} /\|\ln (n+\sigma)\|_{\beta}^{\lambda / s}} \frac{v^{\frac{s \lambda_{1}}{\lambda}-1}}{\prod_{k=1}^{s}\left(v+c_{k}\right)} d v \\
& =O\left(\frac{1}{\|\ln (n+\sigma)\|_{\beta}^{\widetilde{\lambda}_{1}}}\right), \\
\widetilde{K}_{2} & =\frac{\Gamma^{i_{0}}\left(\frac{1}{\alpha}\right) k_{s}\left(\widetilde{\lambda}_{1}\right)}{\alpha^{i_{0}-1} \Gamma\left(\frac{i_{0}}{\alpha}\right)} \in \mathbf{R}_{+} .
\end{aligned}
$$

Proof By Lemma 1, the Hermite-Hadamard inequality (cf. [37]), (10), and (15), it follows that

$$
\begin{aligned}
& w_{\lambda}\left(\lambda_{2}, n\right) \\
& <\int_{\left(\frac{1}{2}, \infty\right)^{i_{0}}} \frac{\|\ln (n+\sigma)\|_{\beta}^{\lambda_{2}}\|\ln (x+\tau)\|_{\alpha}^{\lambda_{1}-i_{0}} d x}{\prod_{k=1}^{s}\left[\|\ln (x+\tau)\|_{\alpha}^{\lambda / s}+c_{k}\|\ln (n+\sigma)\|_{\beta}^{\lambda / s}\right] \prod_{i=1}^{i_{0}}\left(x_{i}+\tau_{i}\right)} \\
& \stackrel{u_{i}=\ln \left(x_{i}+\tau_{i}\right)}{=} \int_{\left\{u \in \mathbf{R}_{+}^{i_{0}} ; u_{i}>\ln \left(\frac{1}{2}+\tau_{i}\right)\right\}} \frac{\|\ln (n+\sigma)\|_{\beta}^{\lambda_{2}}\|u\|_{\alpha}^{\lambda_{1}-i_{0}}}{\prod_{k=1}^{s}\left[\|u\|_{\alpha}^{\lambda / s}+c_{k}\|\ln (n+\sigma)\|_{\beta}^{\lambda / s}\right]} d u \\
& \leq \int_{\mathbf{R}_{+}^{i_{0}}} \frac{\|n-\sigma\|_{\beta}^{\lambda_{2}}\|u\|_{\alpha}^{\lambda_{1}-i_{0}}}{\prod_{k=1}^{s}\left[\|u\|_{\alpha}^{\lambda / s}+c_{k}\|\ln (n+\sigma)\|_{\beta}^{\lambda / s}\right]} d u \\
& =\lim _{M \rightarrow \infty} \int_{\mathbf{D}_{M}} \frac{\|\ln (n+\sigma)\|_{\beta}^{\lambda_{2}} M^{\lambda_{1}-i_{0}}\left[\sum_{i=1}^{j_{0}}\left(\frac{u_{i}}{M}\right)^{\alpha}\right]^{\left(\lambda_{1}-i_{0}\right) / \alpha}}{\prod_{k=1}^{s}\left\{M^{\frac{\lambda}{s}}\left[\sum_{i=1}^{i_{0}}\left(\frac{u_{i}}{M}\right)^{\alpha}\right]^{\frac{\lambda}{\alpha s}}+c_{k}\|\ln (n+\sigma)\|_{\beta}^{\frac{\lambda}{s}}\right\}} d u \\
& =\lim _{M \rightarrow \infty} \frac{M^{i_{0}} \Gamma^{i_{0}}\left(\frac{1}{\alpha}\right)}{\alpha^{i_{0}} \Gamma\left(\frac{i_{0}}{\alpha}\right)} \int_{0}^{1} \frac{\|\ln (n+\sigma)\|_{\beta}^{\lambda_{2}} M^{\lambda_{1}-i_{0}} t^{\left(\lambda_{1}-i_{0}\right) / \alpha} t^{\frac{i_{0}}{\alpha}-1}}{\prod_{k=1}^{s}\left(M^{\frac{\lambda}{s}} t^{\frac{\lambda}{\alpha s}}+c_{k}\|\ln (n+\sigma)\|_{\beta}^{\frac{\lambda}{s}}\right)} d t \\
& =\lim _{M \rightarrow \infty} \frac{M^{\lambda_{1}} \Gamma^{i_{0}}\left(\frac{1}{\alpha}\right)}{\alpha^{i_{0}} \Gamma\left(\frac{i_{0}}{\alpha}\right)} \int_{0}^{1} \frac{\|\ln (n+\sigma)\|_{\beta}^{\lambda_{2}} t^{\frac{\lambda_{1}}{\alpha}-1}}{\prod_{k=1}^{s}\left(M^{\frac{\lambda}{s}} t^{\frac{\lambda}{\alpha s}}+c_{k}\|\ln (n+\sigma)\|_{\beta}^{\frac{\lambda}{s}}\right)} d t
\end{aligned}
$$




$$
\begin{aligned}
& \stackrel{t=\|\ln (n+\sigma)\|_{\beta}^{\alpha} M^{-\alpha} v^{\alpha s / \lambda}}{=} \frac{s \Gamma^{i_{0}}\left(\frac{1}{\alpha}\right)}{\lambda \alpha^{i_{0}-1} \Gamma\left(\frac{i_{0}}{\alpha}\right)} \int_{0}^{\infty} \frac{v^{\frac{s \lambda}{\lambda}-1}}{\prod_{k=1}^{s}\left(v+c_{k}\right)} d v \\
& =\frac{\Gamma^{i_{0}}\left(\frac{1}{\alpha}\right)}{\alpha^{i_{0}-1} \Gamma\left(\frac{i_{0}}{\alpha}\right)} k_{s}\left(\lambda_{1}\right)=K_{2} \text {. }
\end{aligned}
$$

Hence, we have (17). In the same way, we have (18).

By the decreasing property and (10), similarly to the proof of (11), we find

$$
\begin{aligned}
& w_{\lambda}\left(\tilde{\lambda}_{2}, n\right) \\
& >\int_{\left\{x \in \mathbf{R}_{+}^{i_{0}} ; x_{i} \geq e-\tau_{i}\right\}} \frac{\|\ln (n+\sigma)\|_{\beta}^{\tilde{\lambda}_{2}}\|\ln (x+\tau)\|_{\alpha}^{\tilde{\lambda}_{1}-i_{0}} d x}{\prod_{k=1}^{s}\left[\|\ln (x+\tau)\|_{\alpha}^{\lambda / s}+c_{k}\|\ln (n+\sigma)\|_{\beta}^{\lambda / s}\right] \prod_{i=1}^{i_{0}}\left(x_{i}+\tau_{i}\right)} \\
& u_{i}=\ln \left(x_{i}+\tau_{i}\right)\|\ln (n+\sigma)\|_{\beta}^{\tilde{\lambda}_{2}} \int_{\left\{u \in \mathbf{R}_{+}^{i_{0}} ; u_{i} \geq 1\right\}} \frac{\|u\|_{\alpha}^{\tilde{\lambda}_{1}-i_{0}} d u}{\prod_{k=1}^{s}\left[\|u\|_{\alpha}^{\lambda / s}+c_{k}\|\ln (n+\sigma)\|_{\beta}^{\lambda / s}\right]} \\
& =\|\ln (n+\sigma)\|_{\beta}^{\tilde{\lambda}_{2}} \lim _{M \rightarrow \infty} \int \ldots \int_{D_{M}} \frac{\left\{\sum_{i=1}^{i_{0}}\left(\frac{u_{i}}{M}\right)^{\alpha}\right\}^{\frac{\tilde{\lambda}_{1}-i_{0}}{\alpha}} M^{\tilde{\lambda}_{1}-i_{0}} d u_{1} \cdots d u_{i_{0}}}{\prod_{k=1}^{s}\left[\left\{\sum_{i=1}^{i_{0}}\left(\frac{u_{i}}{M}\right)^{\alpha}\right\}^{\frac{\lambda}{\alpha s}} M^{\frac{\lambda}{s}}+c_{k}\|\ln (n+\sigma)\|_{\beta}^{\lambda / s}\right]} \\
& =\frac{M^{i_{0}} \Gamma^{i_{0}}\left(\frac{1}{\alpha}\right)}{\alpha^{i_{0}} \Gamma\left(\frac{i_{0}}{\alpha}\right)}\|\ln (n+\sigma)\|_{\beta}^{\tilde{\lambda}_{2}} \lim _{M \rightarrow \infty} \int_{\frac{i_{0}}{M^{\alpha}}}^{1} \frac{t^{\frac{\tilde{\lambda}_{1}-i_{0}}{\alpha}} M^{\tilde{\lambda}_{1}-i_{0}} t^{\frac{i_{0}}{\alpha}-1}}{\prod_{k=1}^{s}\left[t^{\frac{\lambda}{\alpha s}} M^{\frac{\lambda}{s}}+c_{k}\|\ln (n+\sigma)\|_{\beta}^{\lambda / s}\right]} d t \\
& =\frac{s \Gamma^{i_{0}}\left(\frac{1}{\alpha}\right)}{\lambda \alpha^{i_{0}-1} \Gamma\left(\frac{i_{0}}{\alpha}\right)} \int_{\frac{i_{0}^{\lambda /(\alpha s)}}{\|\ln (n+\sigma)\|_{\beta}^{\lambda / s}}}^{\infty} \frac{v^{\frac{\tilde{s}_{1}}{\lambda}-1} d v}{\prod_{k=1}^{s}\left(v+c_{k}\right)}=\widetilde{K}_{2}\left(1-\widetilde{\theta}_{\lambda}(n)\right)>0, \\
& 0<\tilde{\theta}_{\lambda}(n)=\frac{s}{\lambda k_{s}\left(\widetilde{\lambda}_{1}\right)} \int_{0}^{i_{0}^{\lambda /(\alpha s)} /\|\ln (n+\sigma)\|_{\beta}^{\lambda / s}} \frac{v^{\frac{s \lambda_{1}}{\lambda}-1}}{\prod_{k=1}^{s}\left(v+c_{k}\right)} d v \\
& \leq \frac{s}{\lambda k_{s}\left(\widetilde{\lambda}_{1}\right) \prod_{k=1}^{s} c_{k}} \int_{0}^{i_{0}^{\lambda /(\alpha s)} /\|\ln (n+\sigma)\|_{\beta}^{\lambda / s}} v^{\frac{s \tilde{\lambda}_{1}}{\lambda}-1} d v \\
& =\frac{1}{\widetilde{\lambda}_{1} k_{s}\left(\widetilde{\lambda}_{1}\right) \prod_{k=1}^{s} c_{k}} \frac{\tilde{i}_{0} / \alpha}{\|\ln (n+\sigma)\|_{\beta}^{\tilde{\lambda}_{1}}} .
\end{aligned}
$$

Hence, we have (20) and (21).

\section{Main results and operator expressions}

Setting $\Phi(m):=\prod_{i=1}^{i_{0}}\left(m_{i}+\tau_{i}\right)^{p-1}\|\ln (m+\tau)\|_{\alpha}^{p\left(i_{0}-\lambda_{1}\right)-i_{0}}\left(m \in \mathbf{N}^{i_{0}}\right)$ and $\Psi(n):=\prod_{j=1}^{j_{0}}\left(n_{j}+\right.$ $\left.\sigma_{j}\right)^{q-1}\|\ln (n+\sigma)\|_{\beta}^{q\left(j_{0}-\lambda_{2}\right)-j_{0}}\left(n \in \mathbf{N}^{j_{0}}\right)$, wherefrom

$$
[\Psi(n)]^{1-p}=\prod_{j=1}^{j_{0}}\left(n_{j}+\sigma_{j}\right)^{-1}\|\ln (n+\sigma)\|_{\beta}^{p \lambda_{2}-j_{0}}
$$

we have the following.

Theorem 1 If $s \in \mathbf{N}, 0<\alpha, \beta \leq 1,0<c_{1}<\cdots<c_{s}, 0<\lambda \leq s, 0<\lambda_{1} \leq i_{0}, 0<\lambda_{2} \leq j_{0}, \lambda_{1}+$ $\lambda_{2}=\lambda, \tau \in\left[\frac{1}{2}, 1\right]^{i_{0}}, \sigma \in\left[\frac{1}{2}, 1\right]^{j_{0}}$, then for $p>1, \frac{1}{p}+\frac{1}{q}=1, a_{m}, b_{n} \geq 0,0<\|a\|_{p, \Phi},\|b\|_{q, \Psi}<\infty$, 
we have

$$
\begin{aligned}
I & :=\sum_{n} \sum_{m} \frac{a_{m} b_{n}}{\prod_{k=1}^{s}\left[\|\ln (m+\tau)\|_{\alpha}^{\lambda / s}+c_{k}\|\ln (n+\sigma)\|_{\beta}^{\lambda / s}\right]} \\
& <K_{1}^{\frac{1}{p}} K_{2}^{\frac{1}{q}}\|a\|_{p, \Phi}\|b\|_{q, \Psi},
\end{aligned}
$$

where the constant factor

$$
K_{1}^{\frac{1}{p}} K_{2}^{\frac{1}{q}}=\left[\frac{\Gamma^{j_{0}}\left(\frac{1}{\beta}\right)}{\beta^{j_{0}-1} \Gamma\left(\frac{j_{0}}{\beta}\right)}\right]^{\frac{1}{p}}\left[\frac{\Gamma^{i_{0}}\left(\frac{1}{\alpha}\right)}{\beta^{i_{0}-1} \Gamma\left(\frac{i_{0}}{\alpha}\right)}\right]^{\frac{1}{q}} k_{s}\left(\lambda_{1}\right)
$$

is the best possible $\left(k_{s}\left(\lambda_{1}\right)\right.$ is indicated by $\left.(15)\right)$.

Proof By the Hölder inequality (cf. [37]), we have

$$
\begin{aligned}
I= & \sum_{n} \sum_{m} \frac{1}{\prod_{k=1}^{s}\left[\|\ln (m+\tau)\|_{\alpha}^{\lambda / s}+c_{k}\|\ln (n+\sigma)\|_{\beta}^{\lambda / s}\right]} \\
& \times\left[\frac{\|\ln (m+\tau)\|_{\alpha}^{\left(i_{0}-\lambda_{1}\right) / q}}{\|\ln (n+\sigma)\|_{\beta}^{\left(j_{0}-\lambda_{2}\right) / p}} \frac{\prod_{i=1}^{i_{0}}\left(m_{i}+\tau_{i}\right)^{1 / q}}{\prod_{j=1}^{j_{0}}\left(n_{j}+\sigma_{j}\right)^{1 / p}} a_{m}\right] \\
& \times\left[\frac{\|\ln (n+\sigma)\|_{\beta}^{\left(j_{0}-\lambda_{2}\right) / p}}{\|\ln (m+\tau)\|_{\alpha}^{\left.i_{0}-\lambda_{1}\right) / q}} \frac{\prod_{j=1}^{j_{0}}\left(n_{j}+\sigma_{j}\right)^{1 / p}}{\prod_{i=1}^{i_{0}}\left(m_{i}+\tau_{i}\right)^{1 / q}} b_{n}\right] \\
\leq & \left\{\sum_{m} W_{\lambda}\left(\lambda_{1}, m\right) \prod_{i=1}^{i_{0}}\left(m_{i}+\tau_{i}\right)^{p-1}\|\ln (m+\tau)\|_{\alpha}^{p\left(i_{0}-\lambda_{1}\right)-i_{0}} a_{m}^{p}\right\}^{\frac{1}{p}} \\
& \times\left\{\sum_{n} w_{\lambda}\left(\lambda_{2}, n\right) \prod_{j=1}^{j_{0}}\left(n_{j}+\sigma_{j}\right)^{q-1}\|\ln (n+\sigma)\|_{\beta}^{q\left(j_{0}-\lambda_{2}\right)-j_{0}} b_{n}^{q}\right\}^{\frac{1}{q}} .
\end{aligned}
$$

Then by (17) and (18), we have (23).

For $0<\varepsilon<p \min \left\{\lambda_{1}, 1-\lambda_{2}\right\}, \tilde{\lambda}_{1}=\lambda_{1}-\frac{\varepsilon}{p}, \tilde{\lambda}_{2}=\lambda_{2}+\frac{\varepsilon}{p}$, we set

$$
\begin{aligned}
& \tilde{a}_{m}=\|\ln (m+\tau)\|_{\alpha}^{-i_{0}+\lambda_{1}-\frac{\varepsilon}{p}} \frac{1}{\prod_{i=1}^{i_{0}}\left(m_{i}+\tau_{i}\right)}, \\
& \widetilde{b}_{n}=\|\ln (n+\sigma)\|_{\beta}^{-j_{0}+\lambda_{2}-\frac{\varepsilon}{q}} \frac{1}{\prod_{j=1}^{j_{0}}\left(n_{j}+\sigma_{j}\right)} \quad\left(m \in \mathbf{N}^{i_{0}}, n \in \mathbf{N}^{j_{0}}\right) .
\end{aligned}
$$

Then by (11) and (20)-(22), we obtain

$$
\begin{aligned}
\|\widetilde{a}\|_{p, \Phi}\|\widetilde{b}\|_{q, \Psi}= & \left\{\sum_{m} \prod_{i=1}^{i_{0}}\left(m_{i}+\tau_{i}\right)^{p-1}\|\ln (m+\tau)\|_{\alpha}^{p\left(i_{0}-\lambda_{1}\right)-i_{0}} \widetilde{a}_{m}^{p}\right\}^{\frac{1}{p}} \\
& \times\left\{\sum_{n} \prod_{j=1}^{j_{0}}\left(n_{j}+\sigma_{j}\right)^{q-1}\|\ln (n+\sigma)\|_{\beta}^{q\left(j_{0}-\lambda_{2}\right)-j_{0}} \widetilde{b}_{n}^{q}\right\}^{\frac{1}{q}} \\
= & \left\{\sum_{m}\|\ln (m+\tau)\|_{\alpha}^{-i_{0}-\varepsilon} \frac{1}{\prod_{i=1}^{i_{0}}\left(m_{i}+\tau_{i}\right)}\right\}^{\frac{1}{p}}
\end{aligned}
$$




$$
\begin{gathered}
\times\left\{\sum_{n}\|\ln (n+\sigma)\|_{\beta}^{-j_{0}-\varepsilon} \frac{1}{\prod_{j=1}^{j_{0}}\left(n_{j}+\sigma_{j}\right)}\right\}^{\frac{1}{q}} \\
=\frac{1}{\varepsilon}\left[\frac{\Gamma^{i_{0}}\left(\frac{1}{\alpha}\right)}{i_{0}^{\varepsilon / \alpha} \alpha^{i_{0}-1} \Gamma\left(\frac{i_{0}}{\alpha}\right)}+\varepsilon O(1)\right]^{\frac{1}{p}}\left[\frac{\Gamma^{j_{0}}\left(\frac{1}{\beta}\right)}{j_{0}^{\varepsilon / \beta} \beta^{j_{0}-1} \Gamma\left(\frac{j_{0}}{\beta}\right)}+\varepsilon \widetilde{O}(1)\right]^{\frac{1}{q}} \\
\widetilde{I}:=\sum_{n}\left[\sum_{m} \frac{\widetilde{a}_{m}}{\prod_{k=1}^{s}\left(\|\ln (m+\tau)\|_{\alpha}^{\lambda / s}+c_{k}\|\ln (n+\sigma)\|_{\beta}^{\lambda / s}\right)} \widetilde{b}_{n}\right. \\
=\sum_{n} w_{\lambda}\left(\widetilde{\lambda}_{2}, n\right)\|\ln (n+\sigma)\|_{\beta}^{-j_{0}-\varepsilon} \frac{1}{\prod_{j=1}^{j_{0}}\left(n_{j}+\sigma_{j}\right)} \\
>\widetilde{K}_{2} \sum_{n}\left(1-O\left(\frac{1}{\|\ln (n+\sigma)\|_{\beta}^{\widetilde{\lambda}_{1}}}\right)\right)\|\ln (n+\sigma)\|_{\beta}^{-j_{0}-\varepsilon} \frac{1}{\prod_{j=1}^{j_{0}}\left(n_{j}+\sigma_{j}\right)} \\
=\widetilde{K}_{2}\left[\frac{\Gamma \Gamma^{j_{0}}\left(\frac{1}{\beta}\right)}{\varepsilon j_{0}^{\varepsilon / \beta} \beta^{j_{0}-1} \Gamma\left(\frac{j_{0}}{\beta}\right)}+\widetilde{O}(1)-O(1)\right] .
\end{gathered}
$$

If there exists a constant $K \leq K_{1}^{\frac{1}{p}} K_{2}^{\frac{1}{q}}$, such that (23) is valid when replacing $K_{1}^{\frac{1}{p}} K_{2}^{\frac{1}{q}}$ by $K$, then we have

$$
\begin{aligned}
& \left(K_{2}+o(1)\right)\left[\frac{\Gamma^{j_{0}}\left(\frac{1}{\beta}\right)}{j_{0}^{\varepsilon / \beta} \beta^{j_{0}-1} \Gamma\left(\frac{j_{0}}{\beta}\right)}+\varepsilon \widetilde{O}(1)-\varepsilon O(1)\right] \\
& <\varepsilon \widetilde{I}<\varepsilon K\|\widetilde{a}\|_{p, \varphi}\|\widetilde{b}\|_{q, \psi}=K\left[\frac{\Gamma^{i_{0}}\left(\frac{1}{\alpha}\right)}{i_{0}^{\varepsilon / \alpha} \alpha^{i_{0}-1} \Gamma\left(\frac{i_{0}}{\alpha}\right)}+\varepsilon O(1)\right]^{\frac{1}{p}}\left[\frac{\Gamma^{j_{0}}\left(\frac{1}{\beta}\right)}{j_{0}^{\varepsilon / \beta} \beta^{j_{0}-1} \Gamma\left(\frac{j_{0}}{\beta}\right)}+\varepsilon \widetilde{O}(1)\right]^{\frac{1}{q}} .
\end{aligned}
$$

For $\varepsilon \rightarrow 0^{+}$, we find

$$
\frac{\Gamma^{j_{0}}\left(\frac{1}{\beta}\right) \Gamma^{i_{0}}\left(\frac{1}{\alpha}\right) k_{s}\left(\lambda_{1}\right)}{\beta^{j_{0}-1} \Gamma\left(\frac{j_{0}}{\beta}\right) \alpha^{i_{0}-1} \Gamma\left(\frac{i_{0}}{\alpha}\right)} \leq K\left[\frac{\Gamma^{i_{0}}\left(\frac{1}{\alpha}\right)}{\alpha^{i_{0}-1} \Gamma\left(\frac{i_{0}}{\alpha}\right)}\right]^{\frac{1}{p}}\left[\frac{\Gamma^{j_{0}}\left(\frac{1}{\beta}\right)}{\beta^{j_{0}-1} \Gamma\left(\frac{j_{0}}{\beta}\right)}\right]^{\frac{1}{q}},
$$

and then $K_{1}^{\frac{1}{p}} K_{2}^{\frac{1}{q}} \leq K$. Hence, $K=K_{1}^{\frac{1}{p}} K_{2}^{\frac{1}{q}}$ is the best possible constant factor of (23).

Theorem 2 With the assumptions of Theorem 1 , for $0<\|a\|_{p, \Phi}<\infty$, we have the following inequality with the best constant factor $K_{1}^{\frac{1}{p}} K_{2}^{\frac{1}{q}}$ :

$$
\begin{aligned}
J & :=\left\{\sum_{n}[\Psi(n)]^{1-p}\left(\sum_{m} \frac{a_{m}}{\prod_{k=1}^{s}\left[\|\ln (m+\tau)\|_{\alpha}^{\lambda / s}+c_{k}\|\ln (n+\sigma)\|_{\beta}^{\lambda / s}\right]}\right)^{p}\right\}^{\frac{1}{p}} \\
& <K_{1}^{\frac{1}{p}} K_{2}^{\frac{1}{q}}\|a\|_{p, \Phi},
\end{aligned}
$$

which is equivalent to (23).

Proof We set $b_{n}$ as follows:

$$
b_{n}:=[\Psi(n)]^{1-p}\left(\sum_{m} \frac{a_{m}}{\prod_{k=1}^{s}\left(\|\ln (m+\tau)\|_{\alpha}^{\lambda / s}+c_{k}\|\ln (n+\sigma)\|_{\beta}^{\lambda / s}\right)}\right)^{p-1} .
$$


Then it follows that $J^{p}=\|b\|_{q, \Psi}^{q}$. If $J=0$, then (27) is trivially valid, since $0<\|a\|_{p, \Phi}<\infty$; if $J=\infty$, then it is a contradiction since the right hand side of (27) is finite. Suppose that $0<J<\infty$. Then by (23), we find

$$
\|b\|_{q, \Psi}^{q}=J^{p}=I<K_{1}^{\frac{1}{p}} K_{2}^{\frac{1}{q}}\|a\|_{p, \Phi}\|b\|_{q, \Psi},
$$

namely, $\|b\|_{q, \Psi}^{q-1}=J<K_{1}^{\frac{1}{p}} K_{2}^{\frac{1}{q}}\|a\|_{p, \Phi}$, and then (27) follows.

On the other hand, assuming that (27) is valid, by the Hölder inequality, we have

$$
\begin{aligned}
I= & \sum_{n}(\Psi(n))^{\frac{-1}{q}}\left[\sum_{m} \frac{a_{m}}{\prod_{k=1}^{s}\left(\|\ln (m+\tau)\|_{\alpha}^{\lambda / s}+c_{k}\|\ln (n+\sigma)\|_{\beta}^{\lambda / s}\right)}\right] \\
& \times\left[(\Psi(n))^{\frac{1}{q}} b_{n}\right] \leq J\|b\|_{q, \Psi} .
\end{aligned}
$$

Then by (27), we have (23). Hence (27) and (23) are equivalent.

By the equivalency, the constant factor $K_{1}^{\frac{1}{p}} K_{2}^{\frac{1}{q}}$ in (27) is the best possible. Otherwise, we would reach a contradiction by (28) that the constant factor $K_{1}^{\frac{1}{p}} K_{2}^{\frac{1}{q}}$ in (23) is not the best possible.

For $p>1$, we define two real weight normal discrete spaces $l_{p, \varphi}$ and $l_{q, \psi}$ as follows:

$$
\begin{aligned}
& l_{p, \varphi}:=\left\{a=\left\{a_{m}\right\} ;\|a\|_{p, \Phi}=\left\{\sum_{m} \Phi(m) a_{m}^{p}\right\}^{\frac{1}{p}}<\infty\right\}, \\
& l_{q, \psi}:=\left\{b=\left\{b_{n}\right\} ;\|b\|_{q, \Psi}=\left\{\sum_{n} \Psi(n) b_{n}^{q}\right\}^{\frac{1}{q}}<\infty\right\} .
\end{aligned}
$$

With the assumptions of Theorem 2, in view of $J<K_{1}^{\frac{1}{p}} K_{2}^{\frac{1}{q}}\|a\|_{p, \Phi}$, we have the following definition.

Definition 2 Define a multidimensional Hilbert-type operator $T: l_{p, \Phi} \rightarrow l_{p, \Psi^{1-p}}$ as follows: For $a \in l_{p, \Phi}$, there exists an unique representation $T a \in l_{p, \Psi^{1-p}}$, satisfying for $n \in \mathbf{N}^{j}$,

$$
(T a)(n):=\sum_{m} \frac{a_{m}}{\prod_{k=1}^{s}\left[\|\ln (m+\tau)\|_{\alpha}^{\lambda / s}+c_{k}\|\ln (n+\sigma)\|_{\beta}^{\lambda / s}\right]} .
$$

For $b \in l_{q, \Psi}$, we define the following formal inner product of $T a$ and $b$ as follows:

$$
(T a, b):=\sum_{n} \sum_{m} \frac{a_{m} b_{n}}{\prod_{k=1}^{s}\left[\|\ln (m+\tau)\|_{\alpha}^{\lambda / s}+c_{k}\|\ln (n+\sigma)\|_{\beta}^{\lambda / s}\right]} .
$$

Then by Theorem 1 and Theorem 2, for $0<\|a\|_{p, \varphi},\|b\|_{q, \psi}<\infty$, we have the following equivalent inequalities:

$$
\begin{aligned}
& (T a, b)<K_{1}^{\frac{1}{p}} K_{2}^{\frac{1}{q}}\|a\|_{p, \Phi}\|b\|_{q, \Psi}, \\
& \|T a\|_{p, \Psi 1-p}<K_{1}^{\frac{1}{p}} K_{2}^{\frac{1}{q}}\|a\|_{p, \Phi} .
\end{aligned}
$$


It follows that $T$ is bounded since

$$
\|T\|:=\sup _{a(\neq \theta) \in l_{p, \Phi}} \frac{\|T a\|_{p, \Psi^{1-p}}}{\|a\|_{p, \Phi}} \leq K_{1}^{\frac{1}{p}} K_{2}^{\frac{1}{q}}
$$

Since the constant factor $K_{1}^{\frac{1}{p}} K_{2}^{\frac{1}{q}}$ in (32) is the best possible, we have:

Corollary 1 With the assumptions of Theorem 2, $T$ is defined by Definition 2, it follows that

$$
\|T\|=K_{1}^{\frac{1}{p}} K_{2}^{\frac{1}{q}}=\left[\frac{\Gamma^{j_{0}}\left(\frac{1}{\beta}\right)}{\beta^{j_{0}-1} \Gamma\left(\frac{j_{0}}{\beta}\right)}\right]^{\frac{1}{p}}\left[\frac{\Gamma^{i_{0}}\left(\frac{1}{\alpha}\right)}{\alpha^{i_{0}-1} \Gamma\left(\frac{i_{0}}{\alpha}\right)}\right]^{\frac{1}{q}} k_{s}\left(\lambda_{1}\right) .
$$

Remark 1 (i) Setting $\Phi_{1}(m):=\prod_{i=1}^{i_{0}}\left(m_{i}+1\right)^{p-1}\|\ln (m+1)\|_{\alpha}^{p\left(i_{0}-\lambda_{1}\right)-i_{0}}\left(m \in \mathbf{N}^{i_{0}}\right)$ and $\Psi_{1}(n):=$ $\prod_{j=1}^{j_{0}}\left(n_{j}+1\right)^{q-1}\|\ln (n+1)\|_{\beta}^{q\left(j_{0}-\lambda_{2}\right)-j_{0}}\left(n \in \mathbf{N}^{j_{0}}\right)$, then putting $\tau=\sigma=1$ in (23) and (27), we have the following equivalent inequalities with the best constant factor $K_{1}^{\frac{1}{p}} K_{2}^{\frac{1}{q}}$ :

$$
\begin{aligned}
& \sum_{n} \sum_{m} \frac{a_{m} b_{n}}{\prod_{k=1}^{s}\left[\|\ln (m+1)\|_{\alpha}^{\lambda / s}+c_{k}\|\ln (n+1)\|_{\beta}^{\lambda / s}\right]}<K_{1}^{\frac{1}{p}} K_{2}^{\frac{1}{q}}\|a\|_{p, \Phi_{1}}\|b\|_{q, \Psi_{1}}, \\
& \left\{\sum_{n}\left[\Psi_{1}(n)\right]^{1-p}\left(\sum_{m} \frac{a_{m}}{\prod_{k=1}^{s}\left[\|\ln (m+1)\|_{\alpha}^{\lambda / s}+c_{k}\|\ln (n+1)\|_{\beta}^{\lambda / s}\right]}\right)^{p}\right\}^{\frac{1}{p}} \\
& \quad<K_{1}^{\frac{1}{p}} K_{2}^{\frac{1}{q}}\|a\|_{p, \Phi_{1}} .
\end{aligned}
$$

Hence, (23) and (27) are more accurate inequalities than (35) and (36).

(ii) Putting $i_{0}=j_{0}=1, \lambda=s, \phi_{1}(m):=(m+1)^{p-1} \ln ^{p\left(1-\lambda_{1}\right)-1}(m+1)(m \in \mathbf{N})$ and $\psi_{1}(n):=$ $(n+1)^{q-1} \ln ^{q\left(1-\lambda_{2}\right)-1}(n+1)(n \in \mathbf{N})$, in (32), we have the following new inequality:

$$
\begin{aligned}
\sum_{m=1}^{\infty} & \sum_{n=1}^{\infty} \frac{a_{m} b_{n}}{\prod_{k=1}^{s} \ln (m+1)(n+1)^{c_{k}}} \\
& <\frac{\pi}{\sin \left(\pi \lambda_{1}\right)} \sum_{k=1}^{s} \prod_{j=1}^{s} \frac{c_{k}^{\lambda_{1}-1}}{c_{j}-c_{k}}\|a\|_{p, \phi_{1}}\|b\|_{q, \psi_{1}} .
\end{aligned}
$$

In particular, for $s=c_{k}=1, \lambda_{1}=\frac{1}{q}, \lambda_{2}=\frac{1}{p}$ in (37), we can deduce (4). Hence, (23) is an extension of (4).

\section{Competing interests}

The authors declare that they have no competing interests.

\section{Authors' contributions}

QC participated in the design of the study and performed the numerical analysis. BY carried out the mathematical studies, participated in the sequence alignment and drafted the manuscript. All authors read and approved the final manuscript.

\section{Author details}

${ }^{1}$ Department of Computer Science, Guangdong University of Education, Guangzhou, Guangdong 510303, P.R. China.

2Department of Mathematics, Guangdong University of Education, Guangzhou, Guangdong 510303, P.R. China. 


\section{Acknowledgements}

This work is supported by the National Natural Science Foundation of China (No. 61370186), and 2013 Knowledge Construction Special Foundation Item of Guangdong Institution of Higher Learning College and University (No. 2013KJCX0140).

\section{Received: 17 March 2014 Accepted: 29 July 2014 Published: 22 August 2014}

\section{References}

1. Hardy, GH, Littlewood, JE, Pólya, G: Inequalities. Cambridge University Press, Cambridge (1934)

2. Mitrinović, DS, Pečarić, JE, Fink, AM: Inequalities Involving Functions and Their Integrals and Derivatives. Kluwer Academic, Boston (1991)

3. Yang, BC: Hilbert-Type Integral Inequalities. Bentham Science Publishers, Sharjah (2009)

4. Yang, BC: Discrete Hilbert-Type Inequalities. Bentham Science Publishers, Sharjah (2011)

5. Yang, BC: The Norm of Operator and Hilbert-Type Inequalities. Science Press, Beijing (2009)

6. Yang, BC: Two Types of Multiple Half-Discrete Hilbert-Type Inequalities. Lambert Academic Publishing, Saarbrücken (2012)

7. Yang, BC: On Hilbert's integral inequality. J. Math. Anal. Appl. 220, 778-785 (1998)

8. Yang, BC, Brnetić, I, Krnić, M, Pečarić, JE: Generalization of Hilbert and Hardy-Hilbert integral inequalities. Math. Inequal. Appl. 8(2), 259-272 (2005)

9. Krnić, M, Pečarić, JE: Hilbert's inequalities and their reverses. Publ. Math. (Debr.) 67(3-4), 315-331 (2005)

10. Yang, BC, Rassias, TM: On the way of weight coefficient and research for Hilbert-type inequalities. Math. Inequal. Appl. 6(4), 625-658 (2003)

11. Yang, BC, Rassias, TM: On a Hilbert-type integral inequality in the subinterval and its operator expression. Banach J. Math. Anal. 4(2), 100-110 (2010)

12. Azar, L: On some extensions of Hardy-Hilbert's inequality and applications. J. Inequal. Appl. 2009, Article ID 546829 (2009)

13. Arpad, B, Choonghong, O: Best constant for certain multilinear integral operator. J. Inequal. Appl. 2006, Article ID $28582(2006)$

14. Kuang, JC, Debnath, L: On Hilbert's type inequalities on the weighted Orlicz spaces. Pac. J. Appl. Math. 1(1), 95-103 (2007)

15. Zhong, WY: The Hilbert-type integral inequality with a homogeneous kernel of $-\lambda$-degree. J. Inequal. Appl. 2008 Article ID 917392 (2008)

16. Hong, Y: On Hardy-Hilbert integral inequalities with some parameters. J. Inequal. Pure Appl. Math. 6(4), Art. 92 (2005)

17. Zhong, WY, Yang, BC: On a multiple Hilbert-type integral inequality with the symmetric kernel. J. Inequal. Appl. 2007, Article ID 27962 (2007). doi:10.1155/2007/27962

18. Yang, BC, Krnić, M: On the norm of a multi-dimensional Hilbert-type operator. Sarajevo J. Math. 7(20), 223-243 (2011)

19. Krnić, M, Pečarić, JE, Vuković, P: On some higher-dimensional Hilbert's and Hardy-Hilbert's type integral inequalities with parameters. Math. Inequal. Appl. 11, 701-716 (2008)

20. Krnić, M, Vuković, P: On a multidimensional version of the Hilbert-type inequality. Anal. Math. 38, 291-303 (2012)

21. Adiyasuren, $\mathrm{V}$, Batbold, $\mathrm{T}$ : Some new inequalities similar to Hilbert-type integral inequality with a homogeneous kernel. J. Math. Inequal. 6(2), 183-193 (2012)

22. Adiyasuren, $\mathrm{V}$, Batbold, T, Krnić, M: On several new Hilbert-type inequalities involving means operators. Acta Math Sin. Engl. Ser. 29(8), 1493-1514 (2013)

23. Rassias, MT, Yang, BC: A multidimensional half-discrete Hilbert-type inequality and the Riemann zeta function. Appl. Math. Comput. 225, 263-277 (2013)

24. Li, YJ, He, B: On inequalities of Hilbert's type. Bull. Aust. Math. Soc. 76(1), 1-13 (2007)

25. Yang, BC: A mixed Hilbert-type inequality with a best constant factor. Int. J. Pure Appl. Math. 20(3), 319-328 (2005)

26. Yang, BC: A half-discrete Hilbert-type inequality. J. Guangdong Univ. Educ. 31(3), 1-7 (2011)

27. Zhong, WY: A mixed Hilbert-type inequality and its equivalent forms. J. Guangdong Univ. Educ. 31(5), 18-22 (2011)

28. Zhong, WY: A half discrete Hilbert-type inequality and its equivalent forms. J. Guangdong Univ. Educ. 32(5), 8-12 (2012)

29. Zhong, JH, Yang, BC: On an extension of a more accurate Hilbert-type inequality. J. Zhejiang Univ. Sci. Ed. 35(2), 121-124 (2008)

30. Zhong, JH: Two classes of half-discrete reverse Hilbert-type inequalities with a non-homogeneous kernel J. Guangdong Univ. Educ. 32(5), 11-20 (2012)

31. Zhong, WY, Yang, BC: A best extension of Hilbert inequality involving several parameters. J. Jinan Univ., Nat. Sci. 28(1), 20-23 (2007)

32. Zhong, WY, Yang, BC: A reverse Hilbert's type integral inequality with some parameters and the equivalent forms. Pure Appl. Math. 24(2), 401-407 (2008)

33. Rassias, MT, Yang, BC: On half-discrete Hilbert's inequality. Appl. Math. Comput. 220, 75-93 (2013)

34. Yang, BC, Chen, Q: A half-discrete Hilbert-type inequality with a homogeneous kernel and an extension. J. Inequal. Appl. 2011, 124 (2011). doi:10.1186/1029-242X-2011-124

35. Yang, BC: A half-discrete Hilbert-type inequality with a non-homogeneous kernel and two variables. Mediterr. J. Math. 10(2), 677-692 (2012). doi:10.1007/s00009-012-0213-5

36. Yang, BC: Hilbert-type integral operators: norms and inequalities. In: Pardalos, PM, Georgiev, PG, Srivastava, HM Nonlinear Analysis: Stability, Approximation, and Inequalities, Chapter 42, pp. 771-859. Springer, New York (2012)

37. Kuang, JC: Applied Inequalities. Shangdong Science Technic Press, Jinan (2004)

38. Pan, YL, Wang, HT, Wang, FT: On Complex Functions. Science Press, Beijing (2006)

doi:10.1186/1029-242X-2014-322

Cite this article as: Chen and Yang: On a more accurate multidimensional Mulholland-type inequality. Journal of Inequalities and Applications 2014 2014:322. 